\title{
Organizing Collaboration on Scientific Publications: From Email Lists to Cloud Services
}

\author{
Sönke Bartling
}

Scientific publications-ranging from full papers, abstracts, and presentations to posters, including grant applications and blog posts - are usually written by one or a few authors and then corrected and reviewed by many authors. Collaboration on scientific publications is a cornerstone of science. Many novel tools exist that can be integrated and facilitate this process. However, novel disadvantages come with the changes of the workflow - the future will tell as to which way to work on documents will be the most efficient one.

Here we will discuss several ways of organizing collaboration and discuss the advantages and disadvantages.

The 1.0 way of organizing collaboration:

- Sending out emails with texts, presentations and manual tracking of the version number

- Using the track change or compare documents functionality of word processing tools

- Using data transfer protocols, such as shared local networks or FTP to transfer larger files

- Manual tracking of versions, backups, etc.

Advantage:

- Current state of the art

- Full data control - the process can be organized, so that only known and trusted parties gain access to the content

- Politics-authors and co-authors can decide who sees which version and when

\author{
S. Bartling $(\bowtie)$ \\ German Cancer Research Center, Heidelberg, Germany \\ e-mail: soenkebartling@gmx.de \\ S. Bartling \\ Institute for Clinical Radiology and Nuclear Medicine, Mannheim University Medical \\ Center, Heidelberg University, Mannheim, Germany
}


- Free and open source solutions available

Disadvantage:

- Can be hard to keep track of edits and to integrate changes into a summarized version

- Many "local cultures of keeping track of versions"

- Simultaneous work needs central organization (leading author?) and might create high workload to keep versions in sync

Solutions:

- Standard Email and document processor software packages, FTP, network file systems

- MS Office solutions

- Open Office

- Text editors, LaTex

The cloud way of organizing collaboration:

- Using cloud tools that synchronize folders within the work group and create a version history/backup of old files

- Using collaborative authoring tools to work simultaneously on documents Advantage:

- Simultaneous work

- No out-of-sync version of documents

- Complete history

- No work load overhead for version synchronization and file transfer management

Disadvantages:

- Only $97 \%$ compatibility with current standard word or presentation software solutions

- Third party companies gain access to data and texts, therefore have to be trusted

- Most free solutions are high-maintenance and need high amount of training, local cloud solutions may lack features

- Scepticism regarding novel concepts with both good and bad arguments (the old fashioned way is usually perceived as secure and well established, while disadvantages of novel concepts are over-perceived)

- Collaborative authoring tools do not relieve authors from recursively checking the internal integrity of the documents-low threshold to submit changes may decrease the diligence of contributors 
Solutions (examples):

- Dropbox

- Google documents/drive

- Zotero

- other Concurrent version systems (social coding!) with front ends to focus on collaborative text editing

Open Access This chapter is distributed under the terms of the Creative Commons Attribution Noncommercial License, which permits any noncommercial use, distribution, and reproduction in any medium, provided the original author(s) and source are credited. 